EPJ Web of Conferences 82, 01056 (2015)

DOI: $10.1051 /$ epjconf/20158201056

(C) Owned by the authors, published by EDP Sciences, 2015

\title{
Numerical study of the viscous drop spreading process when transferring heat to a substrate of simple geometry
}

\author{
A.V. Krainov a and G.V. Kuznetsov \\ Tomsk Polytechnic University, 634050 Tomsk, Russia
}

\begin{abstract}
We present a two-dimensional non-stationary model for calculating heat transfer when spreading a liquid viscous drop on a heated substrate. Calculations on the process of fixed drops spreading on a substrate of simple geometry are performed. The hydrodynamic picture of the spreading process is obtained. The influence of the model parameters on the nature of spreading is studied. The temperature profiles of the droplet surface are obtained. The estimation of the time to establish a stationary mode is obtained.
\end{abstract}

Crevice flows of fluid and drops are very common in nature, they are used in different technologies and are promising in terms of new high-intensity heat exchange systems development. Besides the crevice flow on a heat transfer surface, the drop-wise condensation mode can also be realized [1].

Many processes in drops are close to the film processes and processes in horizontal liquid layers [2]. The efficiency of thin films use is determined by low specific liquid consumption and by intensive heat exchange. Many processes can be optimized by thinning the film [3]. Film rupture and the phenomenon of surface dewatering are possible [4].

[5] Describes the regular structures, which can also be regarded as a family of flowing streams, coupled to the contact lines. The presence of an extended thin film can enhance the heat transfer.

The importance of studying the heat transfer in a drop of liquid and its vaporization is determined by the need to improve the spray cooling technology for surfaces [6].

The aim of this work is to study the heat transfer during the spreading of a drop of a viscous liquid on a heated substrate with a simple geometry.

We consider a liquid drop with a constant volume, which lies on a horizontal surface in the gravity field directed perpendicular to the substrate of simple geometry (Fig. 1). Possible physical effects due to chemical and physical processes close to the moving contact line and the phenomenon of contact angle hysteresis are not considered. It is assumed that the contact angle is constant. In addition, the drop is surrounded by a fixed gas, its shape determined by the balance of the capillary and gravitational forces, and its surface is considered to be non-deformable. On the figure 1 the area $\left\{\Omega:=0 \leq r_{1} \leq R\right.$, $\left.0 \leq Z \leq B\left(r_{1}\right)\right\}$ is determined, where $R$ - wetted spot radius, $Z=B\left(r_{1}\right)$ - function which describes the drop surface (all given values in Fig. 1 are dimensional).

The Cartesian coordinate system (plane $X Y$ ) corresponds to the substrate and the axis of the drop symmetry coincides with the axis $0 Z$, gravity vector is directed against the axis $Z$. To study the drop

\footnotetext{
${ }^{a}$ Corresponding author: lux_veritatis@mail.ru
}

This is an Open Access article distributed under the terms of the Creative Commons Attribution License 4.0, which permits unrestricted use, distribution, and reproduction in any medium, provided the original work is properly cited. 


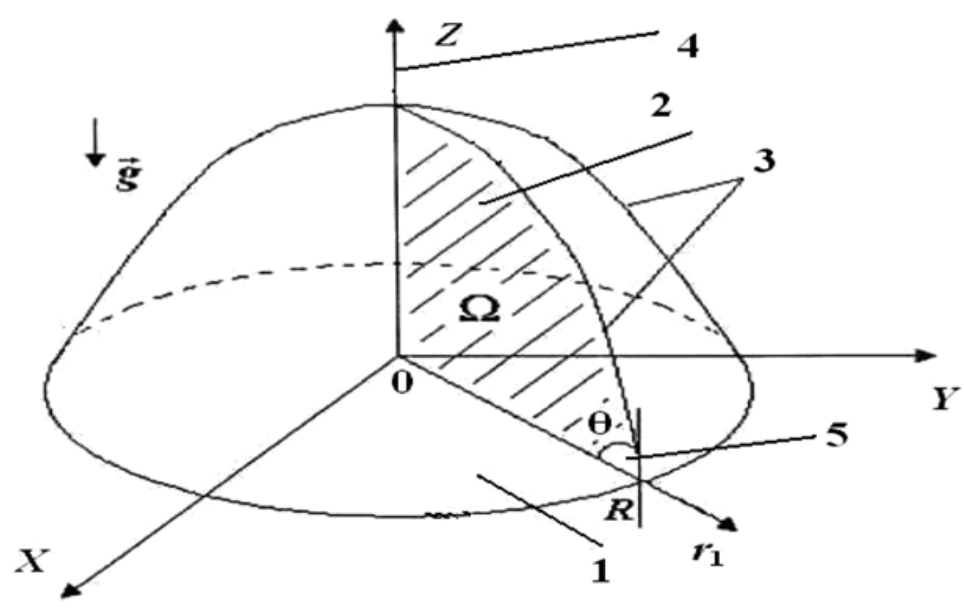

Figure 1. The geometry of the computational region (1-5): 1 - substrate; 2 - the inner region of the drop (hydrodynamic); 3 - the surface of the drop (contact line); 4 - the drop symmetry axis; 5 - wetting angle.

spreading [7] or its deposition on a substrate [8] the equations of energy motion, we used the cylindrical coordinate system (CCS).

Let's write the mathematical formulation of the problem in the CCS in dimensionless form with the corresponding differential equations (discussed above) [9, 10] with the corresponding boundary conditions

$$
\begin{gathered}
\frac{\partial \omega}{\partial \tau}+u_{r} \frac{\partial \omega}{\partial r}+v_{z} \frac{\partial \omega}{\partial z}-\frac{\omega u_{r}}{r}=\operatorname{Pr}\left(\frac{1}{r} \frac{\partial \omega}{\partial r}+\frac{\partial^{2} \omega}{\partial r^{2}}+\frac{\partial^{2} \omega}{\partial z^{2}}-\frac{\omega}{r^{2}}\right)-\operatorname{Ra} \operatorname{Pr} \frac{\partial \theta_{1}}{\partial r} \\
\frac{\partial^{2} \psi}{\partial z^{2}}+\frac{\partial^{2} \psi}{\partial r^{2}}-\frac{1}{r} \frac{\partial \psi}{\partial r}=\omega r \\
\frac{\partial \theta_{1}}{\partial \tau}+u_{r} \frac{\partial \theta_{1}}{\partial r}+v_{z} \frac{\partial \theta_{1}}{\partial z}=\frac{1}{r} \frac{\partial \theta_{1}}{\partial r}+\frac{\partial^{2} \theta_{1}}{\partial r^{2}}+\frac{\partial^{2} \theta_{1}}{\partial z^{2}}
\end{gathered}
$$

Numerical solution of the problems (1)-(3) are conducted in the area $\Omega$ (Fig. 1), limited by the substrate of simple geometry, the surface of the drop and its axis of symmetry. Here Ra - Rayleigh number; $\operatorname{Pr}$ - Prandtl number; $\theta_{1}$ - the dimensionless temperature of the liquid drop; $r, z$ - dimensionless cylindrical coordinates; $u_{r}, v_{z}$ - dimensionless fluid velocity along the corresponding coordinates $r$ and $z$ respectively; $\omega, \psi$ - variables vortex - current function; $\tau$-dimensionless time.

The boundary condition on a substrate when $z=0$ is the condition for adhesion, non-flow and specifies the boundary condition of the second kind. The conditions of non-flow and continuity of heat flow are on the drop symmetry axis.

The boundary conditions on the surface of the drop can be written as [11]

$$
\begin{gathered}
\omega=-\operatorname{Ma} \frac{1}{\sqrt{1+B_{r}^{\prime 2}}}\left(\frac{\partial \theta_{1}}{\partial r}+B_{r}^{\prime} \frac{\partial \theta_{1}}{\partial z}\right), \quad \psi=0 ; \\
-\frac{B_{r}^{\prime}}{\sqrt{1+B_{r}^{\prime 2}}} \frac{\partial \theta_{1}}{\partial r}+\frac{1}{\sqrt{1+B_{r}^{\prime 2}}} \frac{\partial \theta_{1}}{\partial z}=\operatorname{Bi} T .
\end{gathered}
$$


From the kinematic conditions $p_{0}-p+2 \mathrm{Ca} \bar{n} \cdot D \cdot \bar{n}=-2 K$ assuming that $\mathrm{Ca} \ll 1$, we obtain $p-p_{0}=2 K$. The pressure in the fluid is assumed equal to $p=p_{b}-\mathrm{Bo} \cdot z$. Given the mean surface curvature, we obtain the equation for determining the calculated profile of the drop with appropriate boundary conditions (symmetry condition, the condition to set the fixed wetting angle and the condition of the contact line):

$$
\begin{gathered}
\left(\frac{r B^{\prime}}{\sqrt{1+B^{\prime 2}}}\right)_{r}^{\prime}-\operatorname{Bor} B+C r=0, \\
B^{\prime}(0)=0, B^{\prime}\left(R_{1}\right)=-\operatorname{tg} \theta, B\left(R_{1}\right)=0 .
\end{gathered}
$$

The liquid and the substrate material is selected so that for them $\operatorname{tg}^{2} \theta \ll 1$ (water and stainless steel). Then we can assume that $B^{\prime 2^{\prime \prime}} 1$ for any of the current values of the radius $r$ and Eq. (4) can be simplified

$$
\left(r B^{\prime}\right)^{\prime}-\operatorname{Bor} B+C r=0 .
$$

Assuming that the volume of the drop is constant and when integrating the Eq. (6) in the range from 0 to $R_{1}$ we obtain the value of the constant $C$

$$
C=\frac{2 \pi R_{1} \operatorname{tg} \theta+\mathrm{Bo}}{\pi R_{1}^{2}}
$$

Then the resulting function

$$
B(r)=\frac{2 \pi R_{1} \operatorname{tg} \theta+\mathrm{Bo}}{\pi \mathrm{Bo} R_{1}^{2}}+\frac{\operatorname{tg} \theta}{i \sqrt{\mathrm{Bo}} J_{1}\left(i R_{1} \sqrt{\mathrm{Bo}}\right)} J_{0}(i r \sqrt{\mathrm{Bo}})
$$

satisfies the Eq. (6) and the first two conditions (5). Here $J_{0}, J_{1}$ Bessel functions of zero and first order, respectively; $r$-dimensionless value of the drop current radius; $R_{1}$-dimensionless value of the drop radius $\left(0 \leq r<R_{1}\right)$. From the equation (7) taking into account the third condition (5) we obtain an expression of the form

$$
\frac{2 \pi R_{1} \operatorname{tg} \theta+\mathrm{Bo}}{\pi \mathrm{Bo} R_{1}^{2}}+\frac{\operatorname{tg} \theta}{i \sqrt{\mathrm{Bo}} J_{1}\left(i R_{1} \sqrt{\mathrm{Bo}}\right)} J_{0}\left(i R_{1} \sqrt{\mathrm{Bo}}\right)=0 .
$$

Equation (8) (at a fixed contact angle and in the limit Bo $\rightarrow 0$ ) is used for determining the function $R_{1}$ (Bo).

In the boundary conditions, the following notations are taken: $K$ - The mean surface curvature (definable value); $p_{b}$ - The dimensionless pressure at the liquid and solid body boundary; $\mathrm{Ca}$ - capillary number; Ma - Marangoni number; Bo - Bond number.

The equation system of Navier-Stokes in variables "Vortex-Stream Function", energy equations are solved by finite difference method [9]. Difference analogues of the transport equations and the thermal conductivity are solved by the sweep method [10]. The Poisson equation on each sacrificial layer was solved by the successive over-relaxation method. We used the finite-difference scheme of the second order of accuracy.

Calculations were performed on even difference grids. The reliability of the research results was assessed by testing the conservativeness of the used difference grid similar to the method used in [12-16].

The droplet profile and the radius of the wetted spots were calculated numerically, based on the Eq. (4) and boundary conditions (5). Numerical calculations were carried out over a wide range of Bond numbers and the contact wetting angle. The calculations were carried out on a drop of water with volume of $0.11 \mathrm{ml}$, and a typical size of $0.69 \cdot 10^{-2} \mathrm{~m}$. The initial temperature of the substrate was $T_{w}=20^{\circ} \mathrm{C}$. 


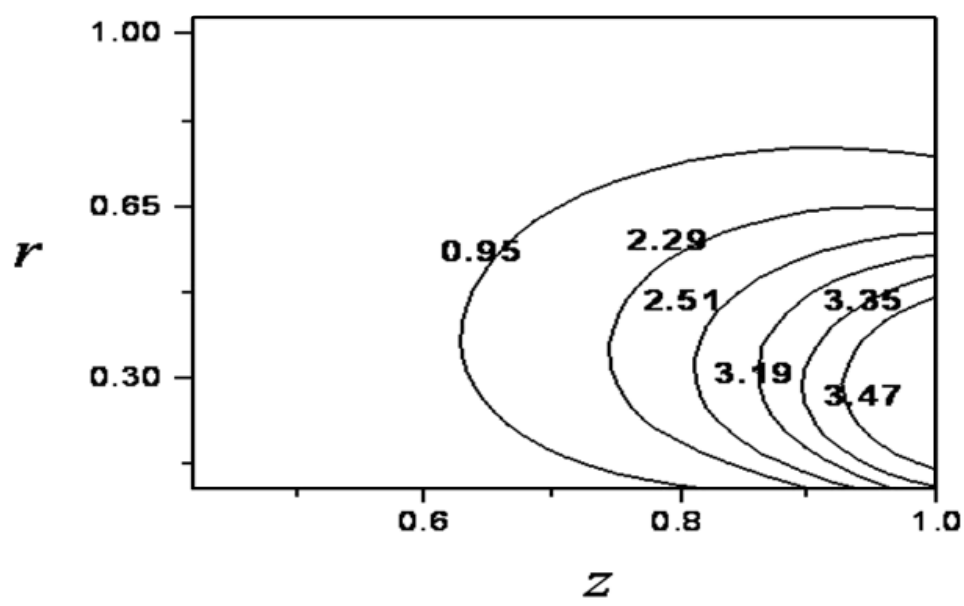

Figure 2. Distribution of stream function at the following numbers $\mathrm{Ma}=277, \mathrm{Ra}=7.1, \operatorname{Pr}=13.7, \mathrm{Bi}=0.55$.

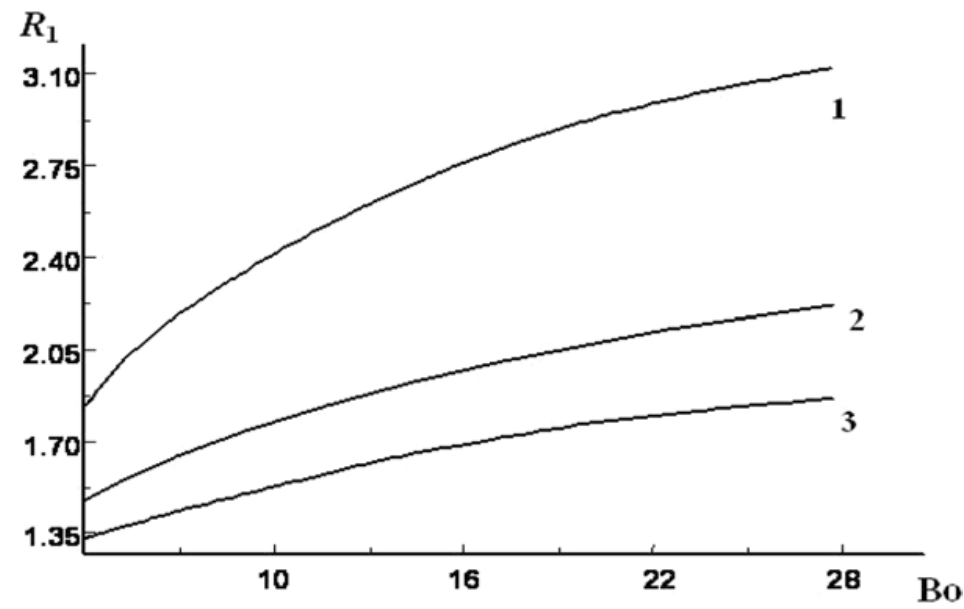

Figure 3. The dependence of the wetted spot radius $R_{1}$ on the Bond number (Bo) at different contact angles: 1) $\theta=4^{\circ}$;2) $\theta=11^{\circ}$;3) $\theta=18^{\circ}$.

Figure 2 shows distribution of stream function at the following numbers $\mathrm{Ma}=277, \mathrm{Ra}=7.1$, $\operatorname{Pr}=13.7, \mathrm{Bi}=0.55$. The maximum stream function falls within $0.27<r<0.39,0.85<z<0.96$, it is when the most intensive formation of vortex structures occur. The calculation results help to reveal the influence of geometric characteristics, heat-exchange conditions on the drop's surface, and capillarygravitational convection on the formation of the stream function field. The distribution pattern of the stream function isolines is qualitatively preserved in the given range of similarity numbers.

Figure 3 shows the dependence of the wetted spot radius on the Bond number for different contact angles: 1) $\theta=4^{\circ}$; 2) $\theta=11^{\circ}$; 3) $\theta=18^{\circ}$. Results were obtained using non-linear Eq. (4) with the conditions (5). Calculations show that the radius of the wetted spots increases with the Bond number. The radius of the drop base increases linearly with increasing gravity at small Bond number values. The nonlinear behavior of the function is observed at high Bond number. Linearity interval increases with the increasing angle $\theta$. 


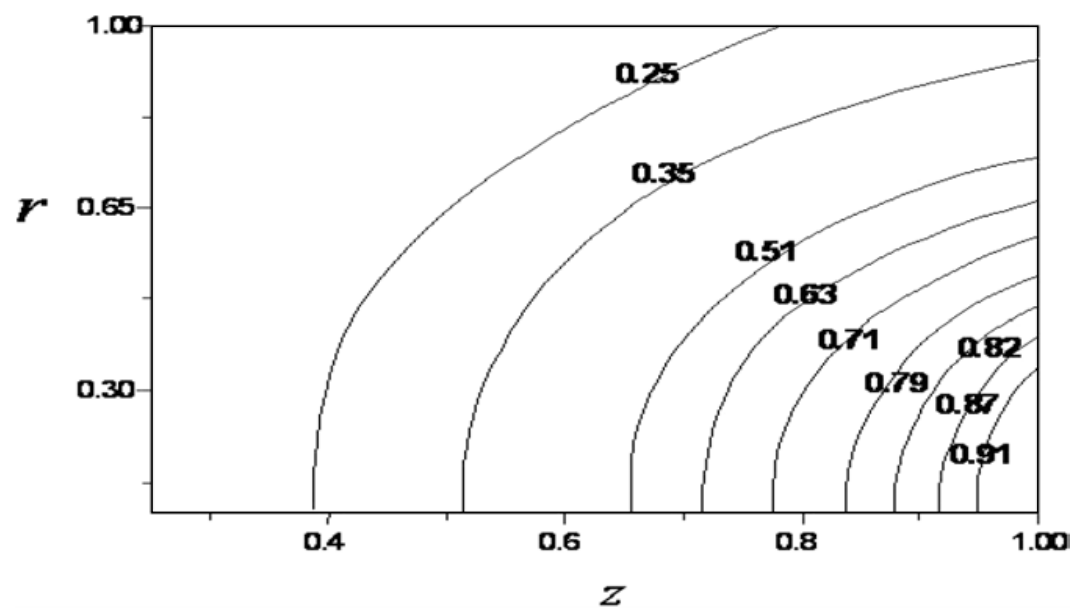

Figure 4. Temperature distribution along the coordinate $r$ for different $z$ values at $\mathrm{Ma}=277, \mathrm{Ra}=7.1, \operatorname{Pr}=13.7$, $\mathrm{Bi}=0.55$.

Figure 4 shows the temperature distribution along the coordinate $r$ for different $\mathrm{z}$ values at $\mathrm{Ma}=$ 277, $\mathrm{Ra}=7.1, \mathrm{Pr}=13.7, \mathrm{Bi}=0.55$. In the context of the considered modes for the drop profile, a single-vortex convective structure develops, formed by thermo-capillary forces. The behavior of the temperature distributions in different drop sections remain the same, qualitatively, with the change of the wetting angle.

Furthermore, as the analysis shows, in comparison with the heat conduction mode (no fluid motion), the presence of convective flow distorts the temperature field.

This study demonstrates the possibility of extending the range of use of the mathematical apparatus [5-8] to solve the conjugate problems of the drop spreading under the gravitational forces on the substrates of a more complex geometry in terms of the heat transfer processes and phase transformations intensification.

\section{References}

[1] V.S. Ajaev, J. Fluid Mechanics 528, 279 (2005)

[2] I.V. Marchuk, J. Engineering Thermophysics 18, 227 (2009)

[3] O.A. Kabov, D.V. Zaitsev, J. Multiphase Science and Technology 21, 249 (2009)

[4] N. Silvi, V. Dussan, J. Physics Fluids 28, 5 (1985)

[5] O.A. Kabov, J. Teplofizika i ae'romehanika 5, 597 (1998)

[6] B. Horacek, K. Kiger, J. Kim, J. Heat and Mass Transfer 48, 1425 (2005)

[7] Y.D. Shikhmurzaev, J. Physics Fluids 9, 266 (1997)

[8] I.A. Guynova, E.I. Sagai`dak, V.N. Popov, J. Sib. zhurn. industr. matem. 7, 36 (2004)

[9] V.M. Paskonov, V.I. Polezhayev, L.A. Chudov, Numerical simulation of heat and mass transfer processes (1984)

[10] A.A. Samarskiy, P.N. Vabischevitch, Computational heat transfer (2003)

[11] V.K. Andreev, Iu.A. Eaponenko, O.N. Eoncharova, V.V. Pukhnachev, Sovremennyè matematicheskie modeli konvektcii (2009)

[12] G.V. Kuznetsov, A.V. Krainov, J. Applied Mechanics and Technical Physics 42, 851 (2001) 
[13] A.V. Krainov, Conjugate heat exchange for a viscous incompressible fluid moving in a rectangular cavity under conditions non-uniformity phase characteristics, Proceedings of International Conference Conjugate problems of mechanics, computer science and ecology, 302 (2004)

[14] G.V. Kuznetsov, A.V. Krainov A.V., J. High Temperature 41, 252 (2003)

[15] Krainov A.V., G.V. Kuznetsov, Conjugate heat transfer during viscous liquid movement in the open cavity, considering its cooling through outer boundary of back surface, EPJ Web of Conferences 76, 1 (2014)

[16] A.V. Kraynov, E.N. Pashkov, A.V. Ponomarev, J. Advanced Materials Research 1040, 876 (2014) 